\title{
Cellular Ubiquity of Calcified Microspheres: A Matter of Degree, Ancient History and the Golgi body?
}

\section{Jean E Aaron}

University of Leeds, Leeds, UK

Corresponding author: Jean E Aaron, Bone Structural Biology Laboratory, School of Biomedical Sciences, Faculty of Biological Sciences, University of Leeds, Leeds, UK, Email: j.e.aaron@leeds.ac.uk

Rec Date: May 09, 2016; Acc Date: June 21, 2016; Pub Date: June 27, 2016

Copyright: (c) 2016 Aaron JE. This is an open-access article distributed under the terms of the Creative Commons Attribution License, which permits unrestricted use, distribution, and reproduction in any medium, provided the original author and source are credited.

Citation: Aaron JE. Cellular Ubiquity of Calcified Microspheres: A Matter of Degree, Ancient History and the Golgi body. J Biomedical Sci. 2016, 5:3.

\section{Abstract}

The following personal viewpoint is a retrospective from diverse histological and elemental microanalytical techniques applied to mineralogenesis that repeatedly suggested a similarity of the inorganic phase of bone to indigenous populations of microbe-like objects $(0.1-1.0 \mu \mathrm{m})$ calcified with phosphate. There is evidence that the microspherical particles are fabricated in the "young" osteocyte golgi apparatus by a process common to unicellular organisms. Inside pre-programmed golgi saccules the "nascent" mineral is contained and controlled by envelopes of specific proteins and lipids in an advancement over a rudimentary version found in calcifying prokaryotes. An intracellular bio-inorganic interface (silicon, calcium and phosphate-rich) is central, primed by carbonate and tempered by trace elements ( $\mathrm{Mg}, \mathrm{Al}, \mathrm{Fe})$. To a lesser extent soft tissues also perform this inherent golgi-directed activity (with potential clinical aberrations). With age the organelle produces calcified microspheres that may be too large (osteoarthritis) or too small (osteoporosis), while "switching off" to allow hard tissues to weaken and "switching on" making soft ones stiffen. Historically the bony biospheres may return to sedimentary rocks to be mined as fertiliser, fuelling an ancient cycle driven by distant golgi bodies with a cargo of bone salt resembling vestigial prokaryotes and primordial "pre-prokaryotes".

Keywords: Calcium phosphate microspheres; Bone substructure; Soft and hard tissue calcification; Golgi apparatus; Silicon and trace elements; Primordial biomineralization

\section{Cellular Ubiquity of Calcified Microspheres}

There is an early etching in Zeitschrift fur Anatomie und Entwicklungsgeschichte of precisely observed soft tissue cells among which are included calcified particles the size of bacteria with less dense centres. Such objects, about 1 micron in diameter, would normally be expected in the hard vertebrate skeleton (and nails), appearing only infrequently elsewhere in abnormal sites of ectopic calcification.

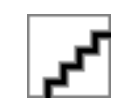

Figure 1 Photomicrographic montage illustrating (top, coloured image) micron-sized mineral particles (red arrow) in arrays of microbe-like, Gram positive-stained chains (crystal violet/ iodine method; Pearse, 1968) in a thin $(3 \mu \mathrm{m})$, undecalcified, cryosection of ox bone. (Light microscope, Nomarski optics; original magnification 1,000X).

Superimposed upon (middle) an unstained ultrathin section of corresponding electron dense objects of similar micron dimension (blue arrow) in bridged assemblies (transmission electron microscope, as a negative image for better definition; original magnification 50,000X), with (bottom, inset) an individual calcified microsphere (white arrow; about $1 \mu \mathrm{m}$ diameter) of variable density (white; negative image) showing typical substructural detail of clusters of beaded filaments ( $5 \mathrm{~nm}$ wide) around a dark electron lucent centre and tail-like extension (TEM, original magnification 55,000X).

As microspheres calcified with phosphate (/carbonate) they populate the collagenous bone matrix (in an independent partnership) as individual mosaic-like domains of variable histochemistry (e.g. some have transitory osteopontin, others contain acid phosphatase or carbonic anhydrase). The particles appear rounded and discrete in new bone (Figure 1), becoming compacted and deformed by mechanical stress in mature regions, sometimes modulating from an amorphous state to a more crystalline one and thereby altering physical properties and resorption signals. The inorganic phase is governed by established rules of physical chemistry, which in isolation from other factors produce myriads of well-documented, small, uniform dense bodies culminating in clusters and sheets of needle- and plate-like crystals [1,2]. However, the outcome alters with the proximity of organic molecules (e.g. noncollagenous proteins such as osteocalcin and bone sialoprotein $[3,4]$ ) added to which is the input of an energetically quixotic biology influential within the confines of the juxtanuclear golgi apparatus, where the basic bone salt may be marshalled and modulated according to extraneous functional demand $[5,6]$. 
In support of such a golgi-directed event in bone (and dentine and keratin) are reports from different parts of the biological spectrum $[7,8]$ of inorganic calcified objects arising via a common, basic golgi process, the classic example being the algal coccoliths modelled within unique vesicular templates to fulfil a highly specific extracellular role. Similarly in the saccules of the "young" osteocyte calcified particles develop sequentially from silicon calcified with carbonate, first mediated as $40 \mathrm{~nm}$ diameter spherical precursor groups of dense nanospheres which grow into microspheres by attachment to the golgi vesicle walls $[5,6]$. It now seems likely that the golgi apparatus of all cells has this inherent capacity to fabricate calcified microspheres, programmed by an ancient common ancestor, the difference between soft and hard tissues being merely a matter of organellar initiation and degree [9]. Thus they feature across the phyla, being prominent in certain invertebrates, for example, in the aquatic protozoan Spirostomum ambiguum their numbers are dictated by whether the organism is free-swimming in water or is tunnelling through the silt [10]. They also appear in bacteria such as the common, oral Corynebacterium matruchotii (as well as various oral streptococcal strains). Here, in the absence of membranous compartments, their precedent nanospheres arise directly in the cytoplasm and expand within the boundary cell wall enclosure, eventually traversing the outer membrane to emerge as chains of microspheres which self-assemble into a protective capsule [11].

In all such examples a dependence upon the presence of silicon seems to be a hallmark of their EDX (energy dispersive $X$ ray spectroscopy) or FEGSEM X-ray elemental microanalysis. Three other typical and apparently anomalous traits are firstly their strong microbial gram positive staining property (Figure 1; crystal violet/ iodine staining of peptidoglycan, perhaps an echo of prokaryote-related ancestry; however, it is not always histochemically specific and can stain collagen). Second is their widely recognised binding of the antibiotic tetracycline to produce a fluorescent complex so specific that it has become a tool in the histomorphometry of bone formation rate $[12,13]$. Third is a differential affinity for routine nucleic acid histological stains (methyl green/ pyronin Y) showing concentrated red RNA stain in microspheres at the discrete calcification front and paler pink stain in the mature matrix beyond (Figure 2; with acellular bone from fish scales exhibiting the same phenomenon). Small amounts of RNA have been identified microanalytically by others in the extracellular matrix in cell-derived vesicles and contaminants. However, much of this widespread affinity between bone salt and RNA stain seems unlikely to be due to the physical presence of organic nucleic acid macromolecules. While it may therefore be an adsorption artefact, it may be a meaningful one, whereby a complementary, naturally occurring "crystalline" bone salt template for nucleic acid is imprinted as a regular feature of the mineral surface $[14,15]$.

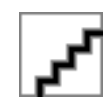

Figure 2 Undecalcified, plastic-embedded section (10 $\mu \mathrm{m}$ thick) of typical cancellous bone stained histologically for nucleic acid (methyl green/ pyronin Y Brachet method) showing relatively strong red staining for RNA at the calcification front (arrowheads) and weaker pink general staining of the calcified matrix (but not the uncalcified osteoid tissue which tends to be light green. Light microscope; original magnification 250X).

For more detailed analysis of the similarities that unite cellfabricated microspheres and the differences that divide them from pure physicochemical precipitates and geological hydroxyapatite the objects can be separated from bone and surrounding tissue [16] using various procedures (digestive chemicals and density fractionation, enzymes, freeze-drying, cryo- or Tema milling and even by exploiting aggressive fungal activity). When relieved of the axial deformation forces the isolated calcified objects mostly return towards their more typical rounded shape, each particle regularly displaying a taillike extension and a heterogeneous composition of clusters of beaded filaments, $5 \mathrm{~nm}$ wide, variable in density (Figure 1). These may coil randomly in ladder-like pairs around a less dense centre (as in bone and bacteria) or may radiate geometrically from it (as in S. ambiguum). There is a frequent predisposition to form one or more superficial bud-like protrusions resulting in interlinked chains of particles and convoluted assemblies (e.g. particularly conspicuous around turkey leg tendon $[17,18]$, Figure 3). In this form the juxtaposed bridged assemblies interconnect to function as a microskeleton of structural complexity that loops around and supports the flexible collagen fibre bundles and is visible under the optical microscope [19]. This postulated microskeleton is apparently less interlinked in the flat bones and more interconnected in the long bones and vertebrae [18], appearing structurally coarse in some anatomical locations and fine in others. As a composite of discrete particles, it is subject to particle slip [20] and the consequent harmless dissipation of microfissures along its boundaries (unless it has transposed to a crystalline state when it is more prone to microfracture). Statistical differences in regional mean size of the calcified microspheres have been reported in relation to anatomical function and are a particular feature in the two major contrasting conditions of skeletal ageing [21] whereby the calcified particles are smaller than normal in osteoporosis $(0.5-$ $0.7 \mu \mathrm{m}$; arising from correspondingly small precursor nanospheres $30-50 \mathrm{~nm}$ ) and larger than normal in osteoarthritis $(0.5-4.0 \mu \mathrm{m}$; arising from large precursor nanospheres $>100 \mathrm{~nm}$ ).

There are distinctions in the proportion of carbonate contained (alleged to be commonplace in the ear ossicles), as well as elemental variations in a range of minor additives including $\mathrm{Mg}$, $\mathrm{Fe}, \mathrm{Al}$ and sometimes $\mathrm{Na}, \mathrm{K}, \mathrm{Cl}, \mathrm{Bo}, \mathrm{Sr}$, some which incorporate into the bone salt lattice increasing or reducing crystallinity (e.g. fluoride and carbonate respectively) and others which adsorb onto its surface. Depending on concentration all 
such additives will individualise behavioural properties (e.g. Fe and $\mathrm{F}$ are beneficial in small amounts, but detrimental in excess). Some of these elemental traces may have implications outside the periosteal and endosteal envelopes in soft tissue systems [18] ranging from connective tissues (e.g., as found in permanent cartilage, intervertebral discs, ligaments, tendons and periosteum) to skeletally unrelated ones (e.g., as found in aorta, trachea). In soft tissues where they are free of compression constraints they tend to be bigger, while smaller particles may be more metabolically active (e.g. aortic calcification is not unusual in fragile, fine-grained osteoporosis where particle-liberating bone resorption is above normal).

Irrespective of their basic nature and hard or soft tissue derivation, when the material around them dies and decays the calcified microspheres remaining have a philosophical permanence about them. Such calcified fragments and bone "dust" (e.g., as produced by the digestive tracts of predatory carnivores or the significant paleohistological activities of soil fungi) may be scattered to the wind or accumulate in a remarkably well-preserved state as geo-biological sedimentary deposits, such as occur in the Khourigba and Senegal phosphorite mines [22]. From this rocky, resting place they are excavated for agriculture as rejuvenating plant fertilisers (sold as bone meal to gardeners).

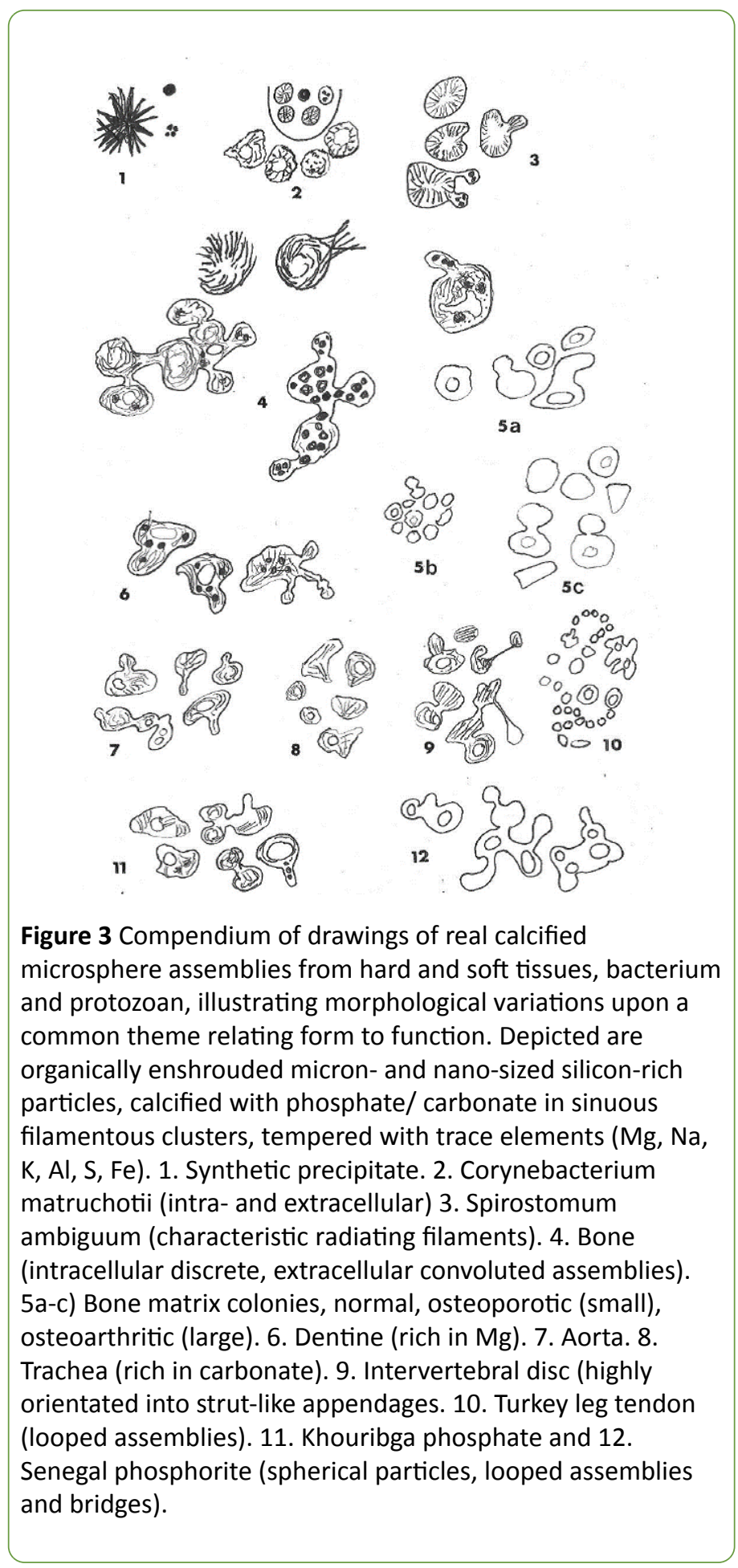

In conclusion, there are calcified, microspherical objects prevalent in bone and dentine, incipient in soft tissues and of a size compatible with dispersion in the circulation. While possessing some physicochemical uniformity there seems to be variety in their dimension, trace elements and interconnection frequency. In general appearance they share with microbes features of form, size and domain-related colonial habit, together with $\mathrm{Si}$ content, gram- and nucleic acid-staining, tetracycline-affinity and the possession of a range of indigenous organic macromolecules, together with a replication capability by budding into chains, as well as functional versatility enabled by trace element "doping". Despite all this, a hypothetical 
microbe-like inorganic phase of bone may seem unlikely and circumstantial, just as the bee orchid is still a flower even if it does look like a bee. Notwithstanding that cautionary proviso, the complex calcified microsphere populations may be interpreted as:

- An entirely physicochemical precipitate of $\mathrm{Ca}^{++} / \mathrm{PO}_{4}--$ in the form of uniform microspheres (initially $<0.1 \mu \mathrm{m}$ in diameter) which grow very slowly and eventually become crystalline sheets? $[1,2,4]$

- Vestigial prokaryotes (like the mitochondrion, centriole, chloroplast) dependent upon the favourable environment of the golgi apparatus to grow rapidly into rounded, subcrystalline objects $>0.1 \mu \mathrm{m}$ ? $[3,6,11,16,23]$

- Primordial "pre-prokaryotes" able to self-assemble rapidly in the confines of the golgi apparatus in groups (each about thirty strong in cross section) of $40 \mathrm{~nm}$ electron dense bodies that may historically pre-date the prokaryote? $[11,12,21]$

Interpretation from static images of dynamic events at a geobiological interface of exceedingly small dimension between inanimate calcified object and animate cell is challenging and the closest to reality could be a cohesion of all three of the above. At the very least there is an unusual assortment of properties to be explained. For the present, it seems that the Greek name apatite, meaning the "deceiver," is especially apt for bone mineral that is fabricated speedily within an unfathomable juxtanuclear golgi-defined space in a daily parade of archaic events that may recapitulate a prebiotic route.

\section{Acknowledgements}

Contributing much of the supporting evidence are the following postdoctoral scientists: Kathryn M. Linton (prokaryote model, age-related disease); Valerie Fallon (bone and protozoan cell culture); Aiman I. Al-Qtaitat (hard and soft tissue comparison); D. Howard Carter (noncollagenous protein immunohistochemistry, bone and dentine substructure) and Philippa E. Garner (osteocyte networks, protozoan model). In addition is $\mathrm{S}$. Mohsen Shahtaheri (osteocalcin immunohistochemistry) and undergraduates Jacqueline Allen (protozoan cell model), Richard Stoker (fish scale bone) and Melanie J.D. Cowdy (Khourigba phosphate microscopy), all supported by technologist Patricia A. Shore, elemental microanalyst Roger C. Shore, microbiologist David G. Adams and clinician Leslie D. Hordon. Inspirational from the beginning with wise counsel was Dr Frederick G.E. Pautard, founding editor of Calcified Tissue Research, Universities of Birmingham, Austin and Leeds, whose main request was to be amazed.

\section{References}

1. Eanes ED, Termine JD, Nylen MU (1973) An electron microscopic study of the formation of amorphous calcium phosphate and its transformation to crystalline apatite. Calcified Tissue Research 12: 143-158.

2. Greenfield DJ, Eanes ED (1972) Formation chemistry of amorphous calcium phosphate prepared from carbonate containing solutions. Calcified Tissue Research 9: 152-162.
3. Carter DH, Scully AJ, Davies RM, Aaron JE (1998) Evidence for phosphoprotein microspheres in bone. Histochem J 30: 677-686.

4. Carter DH, Scully AJ, Heaton DA, Young MP, Aaron JE (2002) Effect of deproteination on bone mineral morphology: implications for biomaterials and aging. Bone 31: 389-395.

5. Fallon V (2006) The Fabrication of Mineral Particles by Bone Cells and Unicellular Organisms. PhD Thesis, University of Leeds.

6. Fallon V, Carter DH, Aaron JE (2014) Mineral fabrication and golgi apparatus activity in the mouse calvarium. Journal of Biomedical Sciences and Engineering 7: 769-779.

7. Pautard FGE (1975) The structure and genesis of calcium phosphate in vertebrates and invertebrates. Colloques | Internationaux CNRS 230: 93-100.

8. Pautard FGE (1981) Calcium phosphate microspheres in biology. Progress in Biological Crystal Growth Characteristics 4: 89-98.

9. Aaron JE, Pautard FGE (1973) A cell cycle in bone mineralization. In The Cell Cycle in Development and Differentiation (Eds. M. Balls and F.S. Billett) Cambridge University Press, Cambridge pp: 325-333.

10. Fallon V, Garner PE, Aaron JE (2016) Stress-induced golgi activity and mineralogenesis: Spirostomum ambiguum, a protozoan model of primordial skeletal behaviour? The International Journal of Developmental Biology.

11. Linton KM, Tapping CR, Adams DG, Carter DH, Shore RC, et al. (2013) A silicon cell cycle in a bacterial model of calcium phosphate mineralogenesis. Micron 44: 419-432.

12. Carter DH, Hatton PV, Aaron JE (1997) The ultrastructure of slamfrozen bone mineral. Histochem J 29: 783-793.

13. Aaron JE, Pautard FGE (1975) Tetracycline staining of bone in normal and pathological states. In Calcium Metabolism, Bone and Metabolic Bone Diseases. Eds. F. Kuhlencordt and H.P. Kruse, Springer-Verlag, Berlin PP: 211-217.

14. Burton FG, Neuman MW, Neuman WF (1969) On the possible role of crystals in the origins of life. I. The adsorption of nucleosides, nucleotides and pyrophosphate by apatite crystals. Curr Mod Biol 3: $20-26$.

15. Burton FG, Neuman MW, Neuman WF (1969) On the possible role of crystals on the origins of life. II. The adsorption of amino acids by apatite crystals. Currents in Modern Biology 3: 69-73.

16. Aaron JE, Oliver B, Clark N, Carter DH (1999) Calcified microspheres as biological entities and their isolation from bone. The Histochemical Journal 31: 455-470.

17. Al-Qtaitat A, Shore RC, Aaron JE (2010) Structural changes in the ageing periosteum using collagen III immuno-staining and chromium labelling as indicators. Journal of Musculoskeletal and Neuronal Interaction 10: 112-123.

18. Al-Qtaitat Al (2007) The Mineral Phase: Calcified Microsphere Populations in Young and Ageing Hard and Soft Tissues. PhD Thesis, University of Leeds.

19. Aaron JE (1980) Demineralization of bone in vivo and in vitro. Evidence for a microskeletal arrangement. Metabolic Bone Disease and Related Research 25: 109-116.

20. Aaron JE (2003) Bone turnover and microdamage. Advances in Osteoporotic Fracture Management 2: 102-110.

21. Linton KM, Hordon LD, Shore RC, Aaron JE (2014) Bone mineral "quality": differing characteristics of calcified microsphere populations at the osteoporotic and osteoarthritic femoral 
articulation front. Journal of Biomedical Science and Engineering 7: 739-755.

22. Pautard FGE (1978) Phosphorus and bone. In New Trends in BioInorganic Chemistry (Eds. R.J.P. Williams, J.R.R.F. Da Silva) Academic Press, London, New York, San Francisco pp: 261-354.
23. Aaron JE, Shore PA (2004) Microscopy and related techniques. In The Physical Measurement of Bone (Eds. C.M. Langton and C.F. Njeh). Institute of Physics Publishing, Philadelphia pp: 225-264. 